\title{
Plant Growth Response of Eight Andean Dry Bean (Phaseolus vulgaris L.) Genotypes to Phosphorus Fertilizer in the Greenhouse
}

\author{
Eninka Mndolwa1 ${ }^{*}$, Harold P. Collins ${ }^{2}$, Phillip N. Miklas ${ }^{3}$ \\ ${ }^{1}$ Department of Crop Science, Sokoine University of Agriculture, Morogoro, Tanzania \\ ${ }^{2}$ United States Department of Agriculture, Agricultural Research Service, Temple, TX, USA \\ ${ }^{3}$ United States Department of Agriculture, Agricultural Research Service, Prosser, WA, USA \\ Email: *eninka.j.mndolwa@gmail.com
}

How to cite this paper: Mndolwa, E., Collins, H.P. and Miklas, P.N. (2018) Plant Growth Response of Eight Andean Dry Bean (Phaseolus vulgaris L.) Genotypes to Phosphorus Fertilizer in the Greenhouse. Agricultural Sciences, 9, 1269-1285. https://doi.org/10.4236/as.2018.910089

Received: August 23, 2018

Accepted: October 26, 2018

Published: October 29, 2018

Copyright $\odot 2018$ by authors and Scientific Research Publishing Inc. This work is licensed under the Creative Commons Attribution International License (CC BY 4.0).

http://creativecommons.org/licenses/by/4.0/

\begin{abstract}
Common bean (Phaseolus vulgaris L.) is an important legume in the tropics, with production limited by low availability of soil phosphorus (P). An experiment was conducted in the glasshouse to evaluate $\mathrm{P}$ use efficiency of eight dry bean genotypes (G122, Montcalm, Taylor Horticulture, Cardinal, Bukoba, Kijivu, Rojo and CAL 143) of Andean origin. The treatments included: no $P$ $\left(0 \mathrm{~kg} \mathrm{P} \mathrm{ha}^{-1}\right)$, normal $\mathrm{P}\left(50 \mathrm{~kg} \mathrm{P} \mathrm{ha}{ }^{-1}\right)$, and high $\mathrm{P}\left(100 \mathrm{~kg} \mathrm{P} \mathrm{ha}^{-1}\right)$. There was variation for the measured traits shoot biomass $(\mathrm{g})$, shoot $\mathrm{P}\left(\mathrm{mg} \mathrm{kg}^{-1}\right)$, root $\mathrm{P}$ $\left(\mathrm{mg} \mathrm{kg}^{-1}\right)$, seed $\mathrm{P}\left(\mathrm{mg} \mathrm{kg}^{-1}\right)$ and seed yield $(\mathrm{g})$ among genotypes and $\mathrm{P}$ treatments. Biomass and all $\mathrm{P}$ concentrations increased with increasing $\mathrm{P}$ level and the genotypes Kijivu, Bukoba, Montcalm and Taylor Horticulture had higher P concentrations than Rojo, G122, Cardinal and CAL 143 across treatments. Genotype $\times$ treatment interactions were observed for shoot biomass. For the no $\mathrm{P}$ treatment, shoot and root biomass were positively correlated with PUE (phosphorus use efficiency). PUE had higher values and varied more among genotypes in the no $\mathrm{P}$ treatment compared to the normal $\mathrm{P}$ and high $\mathrm{P}$ treatments. The results suggest that seed yield in dry bean can be improved by selecting for genotypes with higher PUE under limiting P. The genotypes Bukoba, Kijivu and Montcalm with the highest values for PUE under no $\mathrm{P}$ treatment may be exhibiting some level of tolerance to low soil phosphorus. Higher shoot weight may provide simple criteria for selecting genotypes with greater yield and PUE (phosphorus use efficiency) under limiting P conditions. Therefore, a genotype is desired that can efficiently uptake and utilize available $\mathrm{P}$ under limited availability of this nutrient.
\end{abstract}

\section{Keywords}

Phosphorus Use Efficiency, Phosphorus Susceptibility Index, Phaseolus vulgaris, 
Shoot and Root Dry Weight

\section{Introduction}

Phosphorus $(\mathrm{P})$ deficiency contributes to reduced yield potential of dry bean (Phaseolus vulgaris $\mathrm{L}$.). The most pronounced effect of $\mathrm{P}$ deficiency on plant growth is reduced leaf size, and increased root growth [1]. Accordingly, phosphorus deficiency in dry bean decreases shoot to root biomass ratio but has no effect on the rate of photosynthesis per unit leaf area [2].

Often $\mathrm{P}$ is limiting in tropical soils due to low $\mathrm{pH}$ of the soils and high $\mathrm{P}$ fixation capacity. Correcting $\mathrm{P}$ deficiency is possible through organic or inorganic fertilizer inputs [3]. While applying inorganic fertilizers at low rates can be cost-effective, crop response is often hampered by high $\mathrm{P}$ fixation in the soil. Applying rates of inorganic $P$ fertilizers below the crop requirement will contribute to the continuous depletion of soil $\mathrm{P}$ [3]. Large application of $\mathrm{P}$ fertilizer may be used to circumvent soil depletion but is less effective in tropical soils with low $\mathrm{pH}$ where it is immobile nor is it affordable for subsistence farmers. Therefore, soil $\mathrm{P}$ management is important to insure its availability to crop plants without depleting it from the soil [4].

Several studies indicate that there is variation in $\mathrm{P}$ acquisition efficiency (PAE) and $\mathrm{P}$ use efficiency (PUE) among dry bean genotypes and that the heritability for seed yield under low P supply is high [5] [6] [7]. It is important to understand the mechanisms and genetic control of PAE and PUE in dry bean to facilitate breeding for low $\mathrm{P}$ tolerance [8]. PAE is the ability of a plant to absorb $\mathrm{P}$ from the soil, whereas PUE refers to the plant's ability to make use of the acquired P to produce yield [9] [10]. The genetic control of PUE is complicated because $\mathrm{P}$ is involved in several processes of plant metabolism [11]. Depending on $\mathrm{P}$ availability in the soil, any plant morphological or physiological mechanisms which enhance $\mathrm{P}$ uptake, mobilization, and utilization by the plant will enhance plant tolerance to low $\mathrm{P}$ soils. According to [12] there is variation among dry bean genotypes for PAE and PUE, but PAE is more important for agronomic traits performance. In Ref [13] it reported P-efficient genotypes to have vigorous bean root system.

Improved dry bean cultivars with tolerance to low $\mathrm{P}$ soils may be a viable option to small-scale farmers in the tropics. Alternatively, dry bean cultivars with high PUE will compliment low soil P fertilization by low resource farmers, with minimal access to inorganic $P$ fertilizers. High yielding dry bean cultivars that are tolerant to low $\mathrm{P}$ are not currently available to farmers. There is a need to further identify and characterize dry bean accessions with tolerance to low $\mathrm{P}$ soils. Our objective was to characterize the effect of phosphorus level on vegetative plant growth and phosphorus uptake under limiting soil phosphorus for eight diverse dry bean genotypes. The genotypes are large seeded materials rep- 
resentative of the Andean gene pool [14] and represent parents of existing recombinant inbred mapping populations that could be exploited for genetic studies of low P tolerance. The large seeded Andean dry bean market classes represented by the eight genotypes: red mottled, purple speckled, yellow, red kidney, and cranberry are preferred in Africa, and the latter three types are important in the US.

\section{Materials and Methods}

\subsection{Genetic Materials}

Eight large-seeded Andean dry bean genotypes (Table 1) which represent the parents of five recombinant inbred populations available for advanced genetic studies were tested in a pot study for P efficiency. The G122 (ADP-610) $\times$ Cardinal (ADP-643) and G122 $\times$ Taylor Horticulture (Thort) populations were used to map Beet curly top virus (BCTV) resistance [15] [16]. The ADP indicates an accession within the Andean Diversity Panel (Cichy et al., 2015) which is described in detail on the website (http://arsftfbean.uprm.edu). G122 is a red mottled small cranberry seed type landrace from India with heat tolerance and partial resistance to BCTV. Cardinal is a large seeded cranberry bean cultivar with wide adaptation in the US and with major resistance to BCTV [15]. Taylor Horticulture is an old US cultivar with early maturity but is susceptible to most viruses. The G122 × Montcalm (ADP-636) population was developed for genetic analysis of heat tolerance [17]. Montcalm is a dark red kidney bean cultivar from Michigan State University that is most productive under high inputs. Bukoba (ADP-007) is a yellow seeded "Njano" landrace from Tanzania that is susceptible to terminal drought. Kijivu (ADP-033) is a purpled speckled "Kablanketi" landrace from Tanzania that exhibits drought tolerance. The Rojo (ADP-096) $\times$ CAL 143 (ADP-526) population [18] was developed to map resistance to multiple diseases in Eastern and Southern Africa including bean rust (Uromyces appendiculatus), angular leaf spot (Phaeoisariopsis griseola (Sacc.) Ferr.), and

Table 1. Descriptions for eight dry bean genotypes used in the experiment.

\begin{tabular}{ccccc}
\hline Genotype & ADP\# & Growth habit & Category & Origin \\
\hline G122 & ADP-610 & Bush & Landrace & India \\
Montcalm & ADP-636 & Bush & Cultivar & U.S. \\
Taylor Horticulture & Thort & Bush & Cultivar & U.S. \\
Cardinal & ADP-643 & Bush & Cultivar & U.S. \\
Bukoba & ADP-007 & Bush & Landrace & Tanzania \\
Kijivu & ADP-033 & Bush & Landrace & Tanzania \\
Rojo & ADP-096 & Bush & Cultivar & Tanzania \\
CAL 143 & ADP-526 & Bush & Cultivar & CIACIAT \\
\hline
\end{tabular}

Description of ADP\# can be found at http://arsftfbean.uprm.edu/bean/?page_id=179. 
halo bacterial blight (Pseudomonas syringae pv. phaseolicola). Rojo is a dark red cultivar from Sokoine University of Agriculture (SUA) in Tanzania and CAL 143 is a red mottled "Calima" breeding line from CIAT (Center for Tropical Agriculture) that has been released as Kalima in Malawi and Zambia.

The experiments were conducted in the USDA-ARS glasshouse at Prosser, Washington. Sunshine mix (American Horticultural Supply, Inc., Oxnard, CA, USA), used as the growing media, was analyzed for $\mathrm{P}$ content prior to the experiment to determine suitability for the study. The $\mathrm{P}$ content was $0.40 \mathrm{~g} \mathrm{~kg}^{-1}$, based on the weak acids $\mathrm{P}$ extraction method used (Bray-1 designed for non-calcareous soils). This value is low and determined to be in an inorganic form unavailable for plant use [19]. The clay pots $(20 \mathrm{~cm}$ diameter by $19 \mathrm{~cm}$ depth) were filled with sunshine mix, different MAP rates were incorporated in the sunshine mix of individual pots prior to planting. The three mono-ammonium phosphate (MAP) fertilizer rates were $0 \mathrm{~kg} \mathrm{P} \mathrm{ha}{ }^{-1}(0 \mathrm{ppm}), 50 \mathrm{~kg} \mathrm{P} \mathrm{ha}^{-1}(22.5$ $\mathrm{ppm})$ and $100 \mathrm{~kg} \mathrm{P} \mathrm{ha}{ }^{-1}$ (45 ppm).

Three bean seeds of an individual genotype were planted per pot and later thinned to a single plant per pot about 10 days after planting. Watering was done daily but no additional fertilizers were applied for the duration of the experiment. The day temperature varied between $25^{\circ} \mathrm{C}-28^{\circ} \mathrm{C}$ and night temperature was $23^{\circ} \mathrm{C}$, with a $14 \mathrm{~h}$ day light provided by supplementing natural light with artificial lights.

The experimental design was a RCBD with four replications. The first experiment, with 96 pots ( 8 genotypes $\times 3$ P levels $\times 4$ reps) was harvested at flowering maturity and revealed genotypic differences for different traits. It might be interesting to evaluate shoot, root and seed $\mathrm{P}$ as well. Thus, a second experiment was conducted and expanded to 192 pots to include two harvests one at flowering maturity and another at plant maturity ( 8 genotypes $\times 3$ P levels $\times 4$ replications $\times$ two harvests). This second experiment with the two harvest dates was conducted twice to confirm the findings. So, in effect one partial (Experiment 1) and two full experiments (Experiments 2 and 3 ) were conducted.

The plants from all the pots in experiment 1 and half the pots in experiments 2 and 3 were harvested at flowering maturity. The plant shoots and roots were collected separately, oven dried for $48 \mathrm{~h}$ at $75^{\circ} \mathrm{C}$, and then weighed for biomass (g) on an individual plant basis. The root samples were separated from the growing media by rinsing them with tap water and then patting them dry with paper towels. The dry seed was harvested from the remaining pots in experiments 2 and 3 at harvest maturity.

The dried shoot, root and seed samples were ground using a Wiley mill (Thomas Scientific; Swedesboro, NJ, USA) to pass a $1 \mathrm{~mm}$ sieve. The internal $\mathrm{P}$ concentration $(\mathrm{ppm})$ of the ground tissues $(0.5 \mathrm{~g})$ was determined by inductively coupled plasma mass spectrometry (ICP-MS) using analysis of a nitric acid digest [20]. Shoot P (mg) was calculated as the product of the shoot biomass ( $\mathrm{g}$ ) and shoot $\mathrm{P}\left(\mathrm{mg} \mathrm{g}^{-1}\right)$ on a per plant basis. The $\mathrm{P}$ susceptibility index (PSI) was 
measured using $\left\{\mathrm{PSI}=1-\left[\mathrm{Y}_{1} / \mathrm{Y}\right] / 1-\left[\mathrm{X}_{1} / \mathrm{X}\right]\right\}$; where, $\mathrm{Y}_{1}=$ mean shoot biomass of the low $\mathrm{P}$ treatment for genotype $\mathrm{f}_{1} ; \mathrm{Y}=$ mean shoot biomass of the low $\mathrm{P}$ treatment for all genotypes; $\mathrm{X}_{1}=$ mean shoot biomass of the normal $\mathrm{P}$ treatment for genotype ${ }_{1}$; and $\mathrm{X}=$ mean shoot biomass of the normal $\mathrm{P}$ treatment for all genotypes. Genotype P-use efficiency (PUE) was calculated as biomass yield divided by tissue $\mathrm{P}$ concentration at a given $\mathrm{P}$ rate in the growth media [21] [22], given by; $\mathrm{PUE}=\mathrm{Y}_{1} / \mathrm{P}_{1}$, where, $\mathrm{Y}_{1}=$ Biomass yield on $\mathrm{P}$ level $\mathrm{l}_{1}(\mathrm{~g})$ and $\mathrm{P}_{1}=\mathrm{P}$ concentration on $\mathrm{P}_{\text {level }}$.

\subsection{Data Analysis}

An ANOVA combining data across experiments was used for all measured traits using proc GLM procedure in SAS version 9.4 (2002-2012) SAS Institute, Cary, $\mathrm{NC}$ ). A test of hypotheses for mixed model analysis of variance was performed in which replication was considered random while genotypes and treatments (fertilizer rates) were considered fixed. Mean separation was performed using Tukey $(\mathrm{P} \leq \mathrm{0.05})$. Simple phenotypic correlations were computed among the different trait means for the genotypes in the no P treatment using PROC CORR in SAS.

\section{Results}

\subsection{Phosphorous Response and Genotypic Variability}

There were significant $(P \leq 0.05)$ genotype and treatment effects for all traits measured except genotype for root-P (Table 2). A significant genotype $\times$ treatment

Table 2. Combined analysis of variance for different traits evaluated for eight common bean genotypes in three different experiments.

\begin{tabular}{cccc}
\hline Traits & Source & DF & Mean Square \\
\hline Shoot biomass & Experiment (Exp) & 2 & $1346^{* * *}$ \\
Rep (Exp) & 6 & 17 \\
Treatments & 2 & $737^{* * *}$ \\
Genotypes & 7 & $35^{* *}$ \\
Genotypes $\times$ Exp & 14 & 15 \\
Exp $\times$ Treatments & 4 & $288^{* * *}$ \\
Genotypes $\times$ Treatments & 14 & $22^{*}$ \\
Genotypes $\times$ Exp $\times$ Treatments & 28 & 13 \\
Experiment (Exp) & 2 & $5260^{* * *}$ \\
Rep (Exp) & 6 & $91^{* * *}$ \\
Treatments & 2 & $497^{* * *}$ \\
Genotypes & 7 & $23^{* *}$ \\
Genotypes $\times$ Exp & 14 & $21^{* *}$ \\
\hline
\end{tabular}




\section{Continued}

\begin{tabular}{|c|c|c|c|}
\hline & Exp $\times$ Treatments & 4 & $715^{* * *}$ \\
\hline & Genotypes $\times$ Treatments & 14 & 7 \\
\hline & Genotypes $\times \operatorname{Exp} \times$ Treatments & 28 & 5 \\
\hline \multirow[t]{8}{*}{ Shoot-P } & Experiment (Exp) & 2 & $147,527^{* * *}$ \\
\hline & $\operatorname{Rep}(\operatorname{Exp})$ & 6 & 1284 \\
\hline & Treatments & 2 & $395,028^{* * *}$ \\
\hline & Genotypes & 7 & $7023^{* * *}$ \\
\hline & Genotypes $\times$ Exp & 14 & $3584^{* * *}$ \\
\hline & Exp $\times$ Treatments & 4 & $37,009^{* * *}$ \\
\hline & Genotypes $\times$ Treatments & 14 & 783 \\
\hline & Genotypes $\times \operatorname{Exp} \times$ Treatments & 28 & $1516^{* *}$ \\
\hline \multirow[t]{8}{*}{ Root-P } & Experiment (Exp) & 2 & $113,522^{* * *}$ \\
\hline & $\operatorname{Rep}(\operatorname{Exp})$ & 6 & $14,905^{* * *}$ \\
\hline & Treatments & 2 & $299,358^{\star * *}$ \\
\hline & Genotypes & 7 & 3952 \\
\hline & Genotypes $\times$ Exp & 14 & 2319 \\
\hline & Exp $\times$ Treatments & 4 & $27,241^{* * *}$ \\
\hline & Genotypes $\times$ Treatments & 14 & 2124 \\
\hline & Genotypes $\times \operatorname{Exp} \times$ Treatments & 28 & 1687 \\
\hline \multirow[t]{8}{*}{ Seed-P } & Experiment (Exp) & 1 & 1358 \\
\hline & $\operatorname{Rep}(\operatorname{Exp})$ & 3 & 220 \\
\hline & Treatments & 2 & $5278^{* * *}$ \\
\hline & Genotypes & 7 & $1272^{*}$ \\
\hline & Genotypes $\times$ Exp & 7 & $1332^{*}$ \\
\hline & Exp $\times$ Treatments & 2 & 849 \\
\hline & Genotypes $\times$ Treatments & 14 & 312 \\
\hline & Genotypes $\times \operatorname{Exp} \times$ Treatments & 14 & 457 \\
\hline \multirow[t]{8}{*}{ Seed yield } & Experiment (Exp) & 1 & $2563^{* * *}$ \\
\hline & $\operatorname{Rep}(\operatorname{Exp})$ & 3 & 114 \\
\hline & Treatments & 2 & $631^{* * *}$ \\
\hline & Genotypes & 7 & $447^{\star * *}$ \\
\hline & Genotypes $\times$ Exp & 7 & 119 \\
\hline & Exp $\times$ Treatments & 2 & 100 \\
\hline & Genotypes $\times$ Treatments & 14 & 93 \\
\hline & Genotypes $\times \operatorname{Exp} \times$ Treatments & 14 & 111 \\
\hline
\end{tabular}

${ }^{*},{ }^{* *}$, and ${ }^{* * *}$ represents significance at $0.5,0.01$, and 0.001 levels of probability respectively. Treatments $=$ phosphorous levels. 
effect was only observed for shoot biomass. There were significant genotype $x$ experiment interactions for root biomass, shoot- $\mathrm{P}$ and seed-P, and treatment $\mathrm{x}$ experiment interactions for shoot biomass, root biomass shoot-P and for root-P, but these interactions were due more to differences in magnitude than crossover events. Thus, results focus on examining the significant genotypic, treatment, and genotype $\times$ treatment effects across experiments.

In a combined analysis across treatments and experiments, Montcalm had the highest mean value for shoot biomass while CAL 143 had the lowest. No significant differences were observed among genotypes for root biomass. For shoot-P Thort had the highest mean value while Bukoba had the lowest, root-P did not show any significant differences among genotypes. Thort had the highest value for seed-P while CAL 143 and Kijivu had the lowest. G122 had the highest mean seed yield while Rojo had the lowest (Table 3 ).

Reduced P fertilizer rate ( 0 applied) resulted in reduced shoot biomass, root biomass, shoot-P, root-P, seed-P and seed yield (Figure 1). There was variation between the highest fertilizer treatment $100\left(\mathrm{~kg} \mathrm{P} \mathrm{ha}^{-1}\right)$ with the no $\mathrm{P}$ treatment for all traits measured. The greatest effect was between 0 and 50 with little to no difference between 50 to 100 for shoot biomass, seed yield and seed-P. These results indicate that dry beans are not responsive to more fertilizer $\mathrm{P}$ application at higher level of $\mathrm{P}$ in the soil [23] [24].

Variations have been observed among genotypes and between fertilizer rates for shoot biomass, shoot $\mathrm{P}$ and root $\mathrm{P}$ (Table 4). Overall shoot biomass was the lowest in the no $\mathrm{P}$ treatment compared to 50 and $100 \mathrm{~kg} \mathrm{P} \mathrm{ha}^{-1}$ treatments. For the no P treatment, the genotypes Montcalm, Bukoba and Kijivu had the highest value for shoot biomass while G122 had the lowest. No significant differences

Table 3. Means across treatments and experiments for shoot biomass, root biomass, shoot $\mathrm{P}$, root $\mathrm{P}$, seed $\mathrm{P}$ and seed yield.

\begin{tabular}{cccccccc}
\hline Genotypes & ADP\# & $\begin{array}{c}\text { Shoot } \\
\text { biomass }(\mathrm{g})\end{array}$ & $\begin{array}{c}\text { Root } \\
\text { biomass }(\mathrm{g})\end{array}$ & $\begin{array}{c}\text { Shoot P } \\
\left(\mathrm{mg} \mathrm{kg}^{-1}\right)\end{array}$ & $\begin{array}{c}\text { Root P } \\
\left(\mathrm{mg} \mathrm{kg}^{-1}\right)\end{array}$ & $\begin{array}{c}\text { Seed P } \\
\left(\mathrm{mg} \mathrm{kg}^{-1}\right)\end{array}$ & $\begin{array}{c}\text { Seed } \\
\text { yield }(\mathrm{g})\end{array}$ \\
\hline G122 & ADP-610 & $12^{\mathrm{ab}}$ & $8^{\mathrm{a}}$ & $170^{\mathrm{bc}}$ & $211^{\mathrm{a}}$ & $119^{\mathrm{abc}}$ & $23^{\mathrm{a}}$ \\
Montcalm & ADP-636 & $14^{\mathrm{a}}$ & $10^{\mathrm{a}}$ & $165^{\mathrm{c}}$ & $227^{\mathrm{a}}$ & $116^{\mathrm{bc}}$ & $10^{\mathrm{bc}}$ \\
Taylor Hort & Thort & $12^{\mathrm{ab}}$ & $8^{\mathrm{a}}$ & $208^{\mathrm{a}}$ & $227^{\mathrm{a}}$ & $134^{\mathrm{a}}$ & $16^{\mathrm{ab}}$ \\
Cardinal & ADP-643 & $11^{\mathrm{ab}}$ & $8^{\mathrm{a}}$ & $197^{\mathrm{ab}}$ & $240^{\mathrm{a}}$ & $131^{\mathrm{ab}}$ & $10^{\mathrm{bc}}$ \\
Bukoba & ADP-7 & $13^{\mathrm{a}}$ & $9^{\mathrm{a}}$ & $161^{\mathrm{c}}$ & $217^{\mathrm{a}}$ & $120^{\mathrm{abc}}$ & $10^{\mathrm{bc}}$ \\
Kijivu & ADP-33 & $13^{\mathrm{a}}$ & $9^{\mathrm{a}}$ & $172^{\mathrm{bc}}$ & $211^{\mathrm{a}}$ & $108^{\mathrm{c}}$ & $15^{\mathrm{ab}}$ \\
Rojo & ADP-96 & $12^{\mathrm{ab}}$ & $7^{\mathrm{a}}$ & $183^{\mathrm{abc}}$ & $215^{\mathrm{a}}$ & $126^{\mathrm{ab}}$ & $5^{\mathrm{c}}$ \\
CAL 143 & ADP-526 & $9^{\mathrm{b}}$ & $8^{\mathrm{a}}$ & $181^{\mathrm{abc}}$ & $243^{\mathrm{a}}$ & $107^{\mathrm{c}}$ & $11^{\mathrm{bc}}$ \\
\multicolumn{2}{c}{ Mean } & 12 & 8 & 180 & 224 & 120 & 12 \\
\multicolumn{2}{c}{ CV\% } & 45.7 & 102.3 & 31.6 & 28.4 & 19.1 & 83.6 \\
\hline
\end{tabular}

Means followed by the same or no letter within the same column are not significantly different $(\mathrm{P}=0.05)$ Tukey comparisons of mean. 

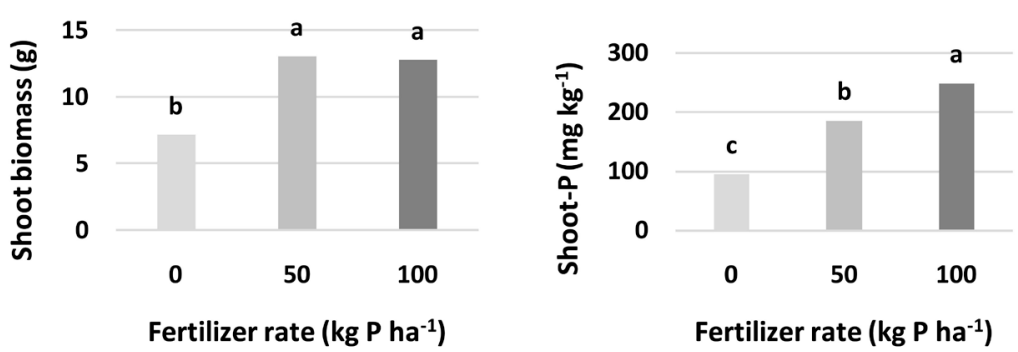

Fertilizer rate (kg P ha-1)
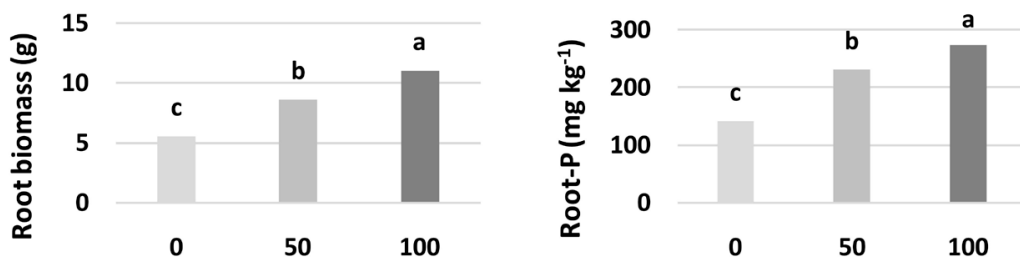

Fertilizer rate $\left(\mathrm{kg} \mathrm{P} \mathrm{ha}^{-1}\right)$

Fertilizer rate ( $\mathrm{kg} \mathrm{P} \mathrm{ha}^{-1}$ )
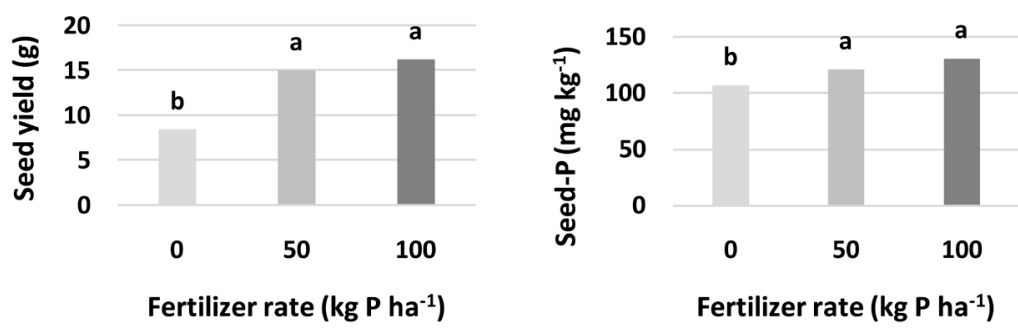

Figure 1. Comparison of treatment means for three $\mathrm{P}$ fertilizer rates $(0,50$, and $100 \mathrm{~kg}$ $\mathrm{ha}^{-1}$ ) combined across eight genotypes and three different greenhouse experiments for shoot biomass, root biomass, shoot $\mathrm{P}$, root $\mathrm{P}$, and two of the three experiments for seed yield, and seed P.

were derived among genotypes and between treatments, for root biomass. For shoot $\mathrm{P}$, variation was observed among genotypes and treatments. In the no $\mathrm{P}$ treatment, Cardinal had the highest value for shoot $\mathrm{P}$ while Bukoba has the lowest. Similarly, variations were observed among genotypes and between treatments for root $\mathrm{P}$. In the no P treatment CAL 143 had the highest value for root $\mathrm{P}$ while Kijivu had the lowest value. For seed P except for CAL 143 in the no P treatment which significantly differed with Taylor Hort in the 50 and $100 \mathrm{~kg} P$ $\mathrm{ha}^{-1}$, all other genotypes and treatments did not differ significantly. For seed yield G122 in the $100 \mathrm{~kg} \mathrm{P} \mathrm{ha}^{-1}$ differed from Montcalm, Cardinal, Bukoba and CAL 143 in the no P treatment. No other significant differences were observed among genotypes within and between treatments (Table 4).

To examine the genotypic effect in different soil treatments, the PSI and PUE values were computed (Table 5 and Table 6).

\subsection{Shoot Biomass}

It has been reported that shoot biomass in dry beans will increase with an increase in soil P levels [25]. Mean separation among fertilizer treatments shows 
that there was no difference between the highest $\left(100 \mathrm{~kg} \mathrm{P} \mathrm{ha}^{-1}\right)$ and normal $\mathrm{P}$ treatments $\left(50 \mathrm{~kg} \mathrm{P} \mathrm{ha}^{-1}\right)$, (Table 4, Figure 1). Shoot biomass was consistently low when no P was added, and the genotypes G122, CAL 143, Cardinal and Rojo were more susceptible to $\mathrm{P}$ deficiency as indicated by significantly lower shoot biomass in no $\mathrm{P}$ treatment than in $50 \mathrm{~kg} \mathrm{P} \mathrm{ha}^{-1}$ and $100 \mathrm{~kg} \mathrm{P} \mathrm{ha}^{-1} \mathrm{P}$ treatments (Table 4).

Table 4. Means for eight bean genotypes grown under three different fertilizer rates $\left(0,50\right.$, and $\left.100 \mathrm{~kg} \mathrm{P} \mathrm{ha}^{-1}\right)$ for shoot biomass, root biomass, shoot $\mathrm{P}$, and root $\mathrm{P}$ (across three experiments), and seed $\mathrm{P}$ and seed yield (across two experiments).

\begin{tabular}{|c|c|c|c|c|c|c|c|c|}
\hline Genotypes & ADP\# & $\begin{array}{c}\text { Rate } \\
\left(\mathrm{kg} \mathrm{P} \mathrm{ha}^{-1}\right)\end{array}$ & $\begin{array}{c}\text { Shoot } \\
\text { biomass (g) }\end{array}$ & $\begin{array}{c}\text { Root } \\
\text { biomass }(\mathrm{g})\end{array}$ & $\begin{array}{c}\text { Shoot P } \\
\left(\mathrm{mg} \mathrm{kg}^{-1}\right)\end{array}$ & $\begin{array}{c}\text { Root P } \\
\left(\mathrm{mg} \mathrm{kg}^{-1}\right)\end{array}$ & $\begin{array}{c}\text { Seed P } \\
\left(\mathrm{mg} \mathrm{kg}^{-1}\right)\end{array}$ & $\begin{array}{c}\text { Seed } \\
\text { yield }(\mathrm{g})\end{array}$ \\
\hline G122 & ADP-610 & 0 & $4^{e}$ & $3^{\mathrm{a}}$ & $95^{\mathrm{cde}}$ & $162^{\text {cdef }}$ & $99^{\mathrm{ab}}$ & $15^{\mathrm{abc}}$ \\
\hline Montcalm & ADP-636 & 0 & $10^{\mathrm{abcde}}$ & $7^{\mathrm{a}}$ & $91^{\mathrm{de}}$ & $146^{\mathrm{def}}$ & $117^{\mathrm{ab}}$ & $1^{\mathrm{b}}$ \\
\hline Taylor Hort & Thort & 0 & $9^{\text {bcde }}$ & $5^{\mathrm{a}}$ & $116^{\text {cde }}$ & $131^{\text {ef }}$ & $107^{\mathrm{ab}}$ & $13^{\mathrm{abc}}$ \\
\hline Cardinal & ADP-643 & 0 & $6^{\text {cde }}$ & $5^{\mathrm{a}}$ & $119^{\text {cde }}$ & $183^{\text {bcdef }}$ & $131^{\mathrm{ab}}$ & $5^{\mathrm{b}}$ \\
\hline Bukoba & ADP-7 & 0 & $10^{\mathrm{abcde}}$ & $5^{\mathrm{a}}$ & $85^{\mathrm{e}}$ & $139 \mathrm{~d}^{\mathrm{ef}}$ & $108^{\mathrm{ab}}$ & $1^{\mathrm{b}}$ \\
\hline Kijivu & ADP-33 & 0 & $10^{\mathrm{abcde}}$ & $6^{\mathrm{a}}$ & $89^{e}$ & $129^{f}$ & $99^{\mathrm{ab}}$ & $20^{\mathrm{abc}}$ \\
\hline Rojo & ADP-96 & 0 & $8^{\text {bcde }}$ & $3^{\mathrm{a}}$ & $102^{\text {cde }}$ & $156^{\text {cdef }}$ & $108^{\mathrm{ab}}$ & $7^{\mathrm{bc}}$ \\
\hline CAL 143 & ADP-526 & 0 & $5^{\mathrm{de}}$ & $5^{\mathrm{a}}$ & $107^{\text {cde }}$ & $165^{\text {cdef }}$ & $87^{\mathrm{b}}$ & $4^{\mathrm{b}}$ \\
\hline G122 & ADP-610 & 50 & $17^{\mathrm{ab}}$ & $9^{\mathrm{a}}$ & $189^{\mathrm{bcd}}$ & $242^{\text {abcde }}$ & $120^{\mathrm{ab}}$ & $26^{\mathrm{ab}}$ \\
\hline Montcalm & ADP-636 & 50 & $18^{\mathrm{a}}$ & $10^{\mathrm{a}}$ & $172^{\text {bcde }}$ & $243^{\mathrm{abcd}}$ & $115^{\mathrm{ab}}$ & $17^{\mathrm{abc}}$ \\
\hline Taylor Hort & Thort & 50 & $15^{\mathrm{abc}}$ & $8^{\mathrm{a}}$ & $223^{\mathrm{ab}}$ & $260^{\mathrm{abc}}$ & $145^{\mathrm{a}}$ & $20^{\mathrm{abc}}$ \\
\hline Cardinal & ADP-643 & 50 & $15^{\mathrm{abc}}$ & $7^{\mathrm{a}}$ & $221^{\mathrm{ab}}$ & $248^{\mathrm{abcd}}$ & $124^{\mathrm{ab}}$ & $10^{\mathrm{abc}}$ \\
\hline Bukoba & ADP-7 & 50 & $13^{\text {abcde }}$ & $7^{\mathrm{a}}$ & $176^{\text {bde }}$ & $231^{\text {abcdef }}$ & $120^{\mathrm{ab}}$ & $14^{\mathrm{abc}}$ \\
\hline Kijivu & ADP-33 & 50 & $16^{\mathrm{ab}}$ & $8^{\mathrm{a}}$ & $173^{\text {bcde }}$ & $238^{\text {abcdef }}$ & $104^{\mathrm{ab}}$ & $15^{\mathrm{abc}}$ \\
\hline Rojo & ADP-96 & 50 & $15^{\mathrm{abcd}}$ & $7^{\mathrm{a}}$ & $191^{\mathrm{bc}}$ & $217^{\mathrm{abcdef}}$ & $126^{\mathrm{ab}}$ & $5^{\mathrm{b}}$ \\
\hline CAL 143 & ADP-526 & 50 & $10^{\mathrm{abcde}}$ & $7^{\mathrm{a}}$ & $192^{\mathrm{bc}}$ & $259^{\mathrm{abc}}$ & $113^{\mathrm{ab}}$ & $13^{\mathrm{abc}}$ \\
\hline G122 & ADP-610 & 100 & $14^{\mathrm{abcd}}$ & $11^{\mathrm{a}}$ & $240^{\mathrm{ab}}$ & $242^{\mathrm{abcd}}$ & $130^{\mathrm{ab}}$ & $30^{\mathrm{a}}$ \\
\hline Montcalm & ADP-636 & 100 & $15^{\mathrm{abcd}}$ & $11^{\mathrm{a}}$ & $245^{\mathrm{ab}}$ & $306^{\mathrm{a}}$ & $125^{\mathrm{ab}}$ & $11^{\mathrm{abc}}$ \\
\hline Taylor Hort & Thort & 100 & $13^{\text {abcde }}$ & $10^{\mathrm{a}}$ & $299^{\mathrm{a}}$ & $305^{\mathrm{a}}$ & $143^{\mathrm{a}}$ & $16^{\mathrm{abc}}$ \\
\hline Cardinal & ADP-643 & 100 & $14^{\mathrm{abcde}}$ & $9^{\mathrm{a}}$ & $266^{\mathrm{ab}}$ & $301^{\mathrm{a}}$ & $137^{\mathrm{ab}}$ & $18^{\mathrm{abc}}$ \\
\hline Bukoba & ADP-7 & 100 & $16^{\mathrm{ab}}$ & $11^{\mathrm{a}}$ & $237^{\mathrm{ab}}$ & $295^{\mathrm{a}}$ & $132^{\mathrm{ab}}$ & $15^{\mathrm{abc}}$ \\
\hline Kijivu & ADP-33 & 100 & $15^{\mathrm{abc}}$ & $11^{\mathrm{a}}$ & $267^{\mathrm{ab}}$ & $281^{\mathrm{ab}}$ & $115^{\mathrm{ab}}$ & $17^{\mathrm{abc}}$ \\
\hline Rojo & ADP-96 & 100 & $14^{\mathrm{abcd}}$ & $10^{\mathrm{a}}$ & $264^{\mathrm{ab}}$ & $283^{\mathrm{ab}}$ & $142^{\mathrm{ab}}$ & $7^{\mathrm{bc}}$ \\
\hline \multirow[t]{3}{*}{ CAL 143} & ADP-526 & 100 & $15^{\mathrm{abc}}$ & $9^{a}$ & $273^{\mathrm{ab}}$ & $319^{\mathrm{a}}$ & $123^{\mathrm{ab}}$ & $17^{\mathrm{abc}}$ \\
\hline & Mean & & 12 & 8 & 180 & 224 & 120 & 12 \\
\hline & CV\% & & 45.7 & 102 & 32 & 28 & 19 & 83.6 \\
\hline
\end{tabular}

Means followed by the same or no letter within the same column are not significantly different $(P=0.05)$ Tukey comparisons of mean. 
Table 5. Phosphorus susceptibility index for shoot biomass (g), and shoot to root biomass ratios for eight bean genotypes grown in three different $\mathrm{P}$ fertilizer rates (low $=0$, normal $=50$, and high $\left.=100 \mathrm{~kg} \mathrm{P} \mathrm{ha}^{-1}\right)$.

\begin{tabular}{cccccc}
\hline \multirow{2}{*}{ Genotype } & \multirow{2}{*}{ ADP\# } & PSI for shoot biomass & \multicolumn{3}{c}{ Shoot to root biomass ratios } \\
\cline { 5 - 6 } & & & $\mathbf{0}\left(\mathrm{kg} \mathrm{P} \mathrm{ha}^{-1)}\right.$ & $\mathbf{5 0}\left(\mathrm{kg} \mathrm{P} \mathrm{ha}^{-1}\right)$ & $\mathbf{1 0 0}\left(\mathrm{kg} \mathrm{P} \mathrm{ha}^{-1}\right)$ \\
\hline G122 & ADP-610 & 1.63 & 1.08 & 1.56 & 1.04 \\
Montcalm & ADP-636 & 0.89 & 1.19 & 1.48 & 1.14 \\
Thort & Thort & 0.94 & 1.28 & 1.55 & 1.07 \\
Cardinal & ADP-643 & 1.19 & 1.09 & 1.88 & 1.16 \\
Bukoba & ADP-7 & 0.49 & 1.54 & 1.5 & 1.14 \\
Kijivu & ADP-33 & 0.85 & 1.26 & 1.53 & 1.17 \\
Rojo & ADP-96 & 0.96 & 1.91 & 1.7 & 1.21 \\
CAL143 & ADP-526 & 0.92 & 1.04 & 1.22 & 1.32 \\
\hline
\end{tabular}

Table 6. Shoot P mg (shoot $\mathrm{P}\left(\mathrm{mg} \mathrm{g}^{-1}\right) \times$ shoot biomass $(\mathrm{g})$ and PUE among varieties.

\begin{tabular}{|c|c|c|c|c|c|c|}
\hline Genotype & ADP\# & $\begin{array}{c}\text { P rate } \\
\left(\mathrm{kg} \mathrm{ha}^{-1}\right)\end{array}$ & $\begin{array}{c}\text { Shoot } \\
\text { biomass (g) }\end{array}$ & $\begin{array}{l}\text { shoot } \mathrm{P} \\
\left(\mathrm{mg} \mathrm{g}^{-1}\right)\end{array}$ & $\begin{array}{c}\text { Shoot P } \\
\text { (mg) }\end{array}$ & $\begin{array}{c}\text { P-use efficiency } \\
\text { (PUE) }\end{array}$ \\
\hline G122 & ADP-610 & 0 & 3.9 & 0.093 & 0.4 & 9.8 \\
\hline Montcalm & ADP-636 & 0 & 9.5 & 0.081 & 0.8 & 11.9 \\
\hline Taylor Hort & Thort & 0 & 8 & 0.112 & 0.9 & 8.9 \\
\hline Cardinal & ADP-643 & 0 & 5.9 & 0.102 & 0.6 & 9.8 \\
\hline Bukoba & ADP-7 & 0 & 9.2 & 0.079 & 0.7 & 13.1 \\
\hline Kijivu & ADP-33 & 0 & 8.7 & 0.086 & 0.7 & 12.4 \\
\hline Rojo & ADP-96 & 0 & 7.1 & 0.092 & 0.7 & 10.1 \\
\hline CAL143 & ADP-526 & 0 & 5.2 & 0.1 & 0.5 & 10.4 \\
\hline G122 & ADP-610 & 50 & 14.8 & 0.196 & 2.9 & 5.1 \\
\hline Montcalm & ADP-636 & 50 & 15.9 & 0.154 & 2.5 & 6.4 \\
\hline Taylor Hort & Thort & 50 & 13.9 & 0.23 & 3.2 & 4.3 \\
\hline Cardinal & ADP-643 & 50 & 12.8 & 0.213 & 2.7 & 4.7 \\
\hline Bukoba & ADP-7 & 50 & 11.8 & 0.17 & 2 & 5.9 \\
\hline Kijivu & ADP-33 & 50 & 14.1 & 0.17 & 2.4 & 5.9 \\
\hline Rojo & ADP-96 & 50 & 12.5 & 0.167 & 2.1 & 6 \\
\hline CAL143 & ADP-526 & 50 & 8.8 & 0.18 & 1.6 & 5.5 \\
\hline G122 & ADP-610 & 100 & 12.5 & 0.248 & 3.1 & 4 \\
\hline Montcalm & ADP-636 & 100 & 13.1 & 0.234 & 3.1 & 4.2 \\
\hline Taylor Hort & Thort & 100 & 11.7 & 0.318 & 3.7 & 3.2 \\
\hline Cardinal & ADP-643 & 100 & 12 & 0.236 & 2.8 & 4.3 \\
\hline Bukoba & ADP-7 & 100 & 13.6 & 0.21 & 2.8 & 4.9 \\
\hline Kijivu & ADP-33 & 100 & 13.7 & 0.255 & 3.5 & 3.9 \\
\hline Rojo & ADP-96 & 100 & 12.3 & 0.243 & 3 & 4.1 \\
\hline CAL143 & ADP-526 & 100 & 13.4 & 0.261 & 3.5 & 3.8 \\
\hline
\end{tabular}




\subsection{Phosphorus Susceptibility Index (PSI)}

The PSI calculations for each genotype are presented in Table 5. Values greater than 1.1 indicate susceptibility to P-deficiency [26]. PSI calculations show that G122 and Cardinal have high PSI values of 1.63 and 1.19 respectively, indicating susceptibility to P-deficiency. These results confirm Tukey means comparisons in Table 4 that G122, Cardinal and Rojo were more susceptible to P deficiencies. The genotype Bukoba had the lowest PSI value of 0.49, suggesting that this genotype may be more tolerant to low P deficiency. Montcalm, Kijivu, CAL143 and Taylor Horticulture have PSI values between 0 and 1.1 indicating moderate to high tolerance to $\mathrm{P}$ deficiency.

\subsection{Root Biomass; Shoot:Root Ratios}

The no-P treatment had lower root biomass compared to normal and high $\mathrm{P}$ treatments (Figure 1). The results for shoot:root ratios are presented in Table 5. We expected the ratio to be higher in low $\mathrm{P}$ treatment compared to high $\mathrm{P}$ treatment. These results follow the trend for the two genotypes Rojo and Bukoba. For all other genotypes the ratio is higher in the normal $\mathrm{P}$ treatment $(50 \mathrm{~kg} \mathrm{P}$ $\mathrm{ha}^{-1}$ ) compared to the low (no-P) and high $\mathrm{P}$ treatment $\left(100 \mathrm{~kg} \mathrm{P} \mathrm{ha}^{-1}\right)$. We speculate that there might be an enhanced root growth response when there was sufficient $\mathrm{P}$ compared to limited or excess $\mathrm{P}$ conditions.

\subsection{Tissue P Concentration}

Tissue $\mathrm{P}$ concentrations allow the identification of $\mathrm{P}$ tolerant genotypes with higher PUE. A genotype that maximizes shoot dry matter while retaining $\mathrm{P}$ is a desired one, because the $P$ retained within the plant can be translocated for seed production. Thus, it was expected that plants with high tissue $\mathrm{P}$ before flowering may have the highest seed production and seeds with higher $\mathrm{P}$ because more $\mathrm{P}$ is available from the vegetative tissue to be mobilized for seed production. Shoot $\mathrm{P}$ concentrations increased with the increasing applied $\mathrm{P}$. The results for $\mathrm{P}$ concentration $\left(\mathrm{mg} \mathrm{kg}^{-1}\right)$ and $\mathrm{P}$ content $(\mathrm{mg})$ are shown in Table 5.

\subsection{Shoot $P$ concentration}

For the no $\mathrm{P}$ treatment, Cardinal had the highest shoot $\mathrm{P}$ concentration of 119 $\mathrm{mg} \mathrm{kg}{ }^{-1}$ while Bukoba had the lowest value of $85 \mathrm{mg} \mathrm{kg}^{-1}$. In the high $\mathrm{P}$ treatment (100 kg P ha ${ }^{-1}$ ), Thort had the highest value of $299 \mathrm{mg} \mathrm{kg}^{-1}$ and Bukoba had the lowest value of $237 \mathrm{mg} \mathrm{kg}^{-1}$ (Table 3). All plants under the $50 \mathrm{~kg} \mathrm{ha}^{-1} \mathrm{P}$ treatment doubled the amount of tissue $\mathrm{P}$ relative to plants under the no- $\mathrm{P}$ treatment that was not reflected in shoot biomass.

Shoot $\mathrm{P}$ concentrations are known to decrease with increasing nutrient deficiency and crop age [27]. The percent $\mathrm{P}$ in shoots (g P $100 \mathrm{~g}$ shoot tissue ${ }^{-1}$ ) for optimal growth is considered to be in the range of $0.3-0.5 \mathrm{~g} \mathrm{P} 100 \mathrm{~g}$ shoot tissue $^{-1}$ during vegetative growth [28]. A $1 \mathrm{~g} \mathrm{P} 100 \mathrm{~g}$ shoot tissue ${ }^{-1}$ concentration or 
higher is considered toxic for plant metabolism. However, this value depends on the crop in question. Most tropical legumes are very sensitive to excess $\mathrm{P}$, as toxicity may occur at much lower levels of $\mathrm{P}$ in the shoot, for example toxicity may occur at $0.3 \%-0.4 \%$ in pigeon pea [11]. Conversely, with decreasing $\mathrm{P}$ supply, $\mathrm{P}$ concentrations in shoots exhibited severe $\mathrm{P}$ deficiency (Table 5). The range of mean shoot $\mathrm{P}$ concentrations under the no $\mathrm{P}$ treatment across varieties ranged from $79.16 \mathrm{mg} \mathrm{kg}^{-1}$ for Bukoba to $112.38 \mathrm{mg} \mathrm{kg}^{-1}$ for Thort, which are equivalent to 0.0008 to $0.0011 \mathrm{~g} \mathrm{P} 100 \mathrm{~g}$ shoot tissue ${ }^{-1}$ respectively. These levels indicate that all genotypes in the no $\mathrm{P}$ treatment had severe deficient shoot $\mathrm{P}$ content compared to the genotypes amended with 50 and $100 \mathrm{~kg} \mathrm{P} \mathrm{ha}^{-1}$.

\subsection{Root P Concentration}

Cardinal had the highest root $\mathrm{P}$ concentration of $183 \mathrm{mg} \mathrm{kg}^{-1}$ while Kijivu had the lowest value of $129 \mathrm{mg} \mathrm{kg}^{-1}$ under the no $\mathrm{P}$ treatment. In the $50 \mathrm{~kg} \mathrm{P} \mathrm{ha}^{-1}$ Thort had the highest value of $260 \mathrm{mg} \mathrm{kg}^{-1}$ and Rojo had the lowest value of 217 $\mathrm{mg} \mathrm{kg}^{-1}$. These results indicate that shoot internal $\mathrm{P}$ and, root internal $\mathrm{P}$ concentrations doubled the amount of tissue $\mathrm{P}$ in $50 \mathrm{~kg} \mathrm{ha}^{-1} \mathrm{P}$ treatments compared to the no $\mathrm{P}$ treatment (Table 3).

\subsection{Seed P Concentration}

Considering the no $\mathrm{P}$ treatment, the genotype Cardinal had higher value for seed P content while CAL 143 had the lowest value. Results suggest that the high value of seed $\mathrm{P}$ for Cardinal may confer an early vigor growth advantage. This early vigor may continue to have a positive effect in later stages as a variety experiences P-deficiency stress.

\subsection{Seed Yield}

Genotypes response to different $\mathrm{P}$ treatments depends on genetic makeup and the level of stress imposed. Kijivu had the highest seed yield under no P treatment followed by G122 and Taylor Horticulture. Although not in the same order, the same three genotypes are among the highest yielding in the normal $\mathrm{P}$ treatment $\left(50 \mathrm{~kg} \mathrm{P} \mathrm{ha}^{-1}\right)$. Overall seed yield was lower in no $\mathrm{P}$ treatment compared to the normal and high $\mathrm{P}$ treatments (Figure 1).

\subsection{Phosphorus Use Efficiency (PUE)}

Results for PUE are presented in Table 6. PUE was calculated as the mean shoot biomass (g) divided by the mean tissue P (mg). A high PUE score reflects a high shoot biomass obtained by remobilizing a high proportion of $\mathrm{P}$. Considering that tissue $\mathrm{P}$ content is later translocated for seed production, a high PUE may or may not be desired for the sake of seed production.

Results show that when no $\mathrm{P}$ is added, the genotype Bukoba had the highest PUE while Thort, G122 and Cardinal had the lowest. Under $50 \mathrm{~kg} \mathrm{ha}^{-1} \mathrm{P}$ treatments, Montcalm exhibited the highest PUE while Thort had the lowest. The 
genotypes Bukoba and Montcalm may be exhibiting tolerance by efficient reallocation of their tissue P. PUE was of no consequence when P was oversupplied above adequate levels to support proper nutrition (Table 6).

\subsection{Correlation Study}

Pearson correlation analysis was used to identify relationships among traits in the no $\mathrm{P}$ treatment (Table 7). A significant correlation at $\mathrm{P} \leq 0.001$ was observed for shoot biomass to PUE, and mean seed yield to mean seed P, suggesting that genotypes with high shoot biomass can utilize $\mathrm{P}$ efficiently when this nutrient element is limited. Other traits with significant correlations at $\mathrm{P} \leq 0.01$ include shoot biomass and root biomass, shoot biomass and shoot $\mathrm{P}$, and shoot $\mathrm{P}$ to root $\mathrm{P}$ concentration, which indicated that shoot and root biomass and internal $\mathrm{P}$ concentrations are associated when beans were grown in $\mathrm{P}$ deficient soils.

Results showed a significant correlation at $\mathrm{P} \leq 0.05$ for several traits: root biomass to PUE, root biomass to shoot $\mathrm{P}$, root biomass to root $\mathrm{P}$, seed $\mathrm{P}$ to shoot $\mathrm{P}, \mathrm{PUE}$ to shoot $\mathrm{P}$ and shoot $\mathrm{P}$ to root $\mathrm{P}$; suggesting a high root biomass was advantageous as there would be greater root surface area during development that leads to higher $\mathrm{P}$ uptake, as represented by high shoot $\mathrm{P}$ and root $\mathrm{P}$. A significant and negative correlation at $\mathrm{P} \leq 0.01$ was observed for the following traits: shoot biomass to PSI shoot, root biomass to PSI shoot, root biomass to PSI root, shoot P concentration to PUE, PUE to PSI shoot, PSI shoot to shoot P and PSI root to root $\mathrm{P}$. These traits negatively correlated with PSI because lower PSI values indicate greater tolerance to P-deficiency. Shoot P concentration and PSI shoot were correlated negatively to PUE, suggesting these two traits are associated with $\mathrm{P}$-deficiency tolerance. The negative correlation between shoot $\mathrm{P}$ concentration

Table 7. Pearson correlations analysis for eight common bean genotypes under no phosphorous treatment.

\begin{tabular}{|c|c|c|c|c|c|c|c|c|c|c|c|}
\hline & $\begin{array}{c}\text { Shoot } \\
\text { biomass (g) }\end{array}$ & $\begin{array}{c}\text { Root dry } \\
\text { weight }(\mathrm{g})\end{array}$ & $\begin{array}{l}\text { Shoot P } \\
\left(\mathrm{mg} \mathrm{kg}^{-1}\right)\end{array}$ & $\begin{array}{c}\text { Root P } \\
\left(\mathrm{mg} \mathrm{kg}^{-1}\right)\end{array}$ & $\begin{array}{c}\text { Seed P } \\
\left(\mathrm{mg} \mathrm{kg}^{-1}\right)\end{array}$ & $\begin{array}{l}\text { Mean seed } \\
\text { yield (g) }\end{array}$ & $\begin{array}{c}\text { Mean seed } \\
\text { P (mg) }\end{array}$ & PUE & $\begin{array}{c}\text { PSI } \\
\text { shoot }\end{array}$ & $\begin{array}{l}\text { PSI } \\
\text { root }\end{array}$ & $\begin{array}{c}\text { shoot P } \\
\text { (mg) }\end{array}$ \\
\hline Root dry wt (g) & $0.80^{* *}$ & 1 & & & & & & & & & \\
\hline Shoot $P$ conc $\left(\mathrm{mg} \mathrm{kg}^{-1}\right)$ & -0.49 & -0.3 & 1 & & & & & & & & \\
\hline Root $P$ conc $\left(\mathrm{mg} \mathrm{kg}^{-1}\right)$ & -0.26 & 0.1 & $0.83^{* *}$ & 1 & & & & & & & \\
\hline Seed $\mathrm{P}$ conc $\left(\mathrm{mg} \mathrm{kg}^{-1}\right)$ & 0.65 & 0.55 & -0.05 & 0.22 & 1 & & & & & & \\
\hline Mean seed yield (g) & -0.12 & -0.05 & 0.16 & -0.07 & -0.39 & 1 & & & & & \\
\hline Mean seed $P(\mathrm{mg})$ & -0.05 & -0.002 & 0.23 & 0.01 & -0.29 & $0.99 * * *$ & 1 & & & & \\
\hline P-use efficiency PUE & $0.95^{\star * *}$ & $0.74^{*}$ & $-0.73^{*}$ & -0.48 & 0.53 & -0.17 & -0.15 & 1 & & & \\
\hline PSI shoot & $-0.80^{*}$ & $-0.54^{*}$ & 0.38 & 0.31 & -0.36 & 0.35 & 0.29 & $-0.77^{*}$ & 1 & & \\
\hline PSI root & -0.62 & $-0.78^{*}$ & 0.08 & -0.12 & -0.54 & 0.30 & 0.23 & -0.53 & 0.67 & 1 & \\
\hline shoot P (mg) & $0.87^{\star *}$ & $0.72^{*}$ & 0.002 & 0.15 & $0.71^{*}$ & -0.01 & 0.11 & $0.68^{*}$ & $-0.69^{*}$ & -0.63 & 1 \\
\hline $\operatorname{root} P(\mathrm{mg})$ & 0.60 & $0.91^{* *}$ & 0.08 & 0.5 & 0.6 & -0.09 & -0.02 & 0.46 & -0.35 & $-0.71^{*}$ & $0.71^{*}$ \\
\hline
\end{tabular}

*,**,***Significant at $\mathrm{P} \leq 0.05,0.01$, or 0.001 , respective. 
and PUE occurs because shoot $\mathrm{P}$ concentration is used to calculate shoot $\mathrm{P}$ which is then used to calculate PUE.

\section{Discussion}

The genotypes used in this study showed variability for shoot biomass, root biomass, shoot $\mathrm{P}$, root $\mathrm{P}$, seed $\mathrm{P}$, and seed yield in response to $\mathrm{P}$ levels. This variability for the above traits indicated differences among genotypes in response to phosphorus availability and supports the importance of $\mathrm{P}$ as a nutrient for common bean production [29]. Among other traits, shoot and root biomass were correlated with PUE in this study and are important traits for low $\mathrm{P}$ tolerance evaluation in common beans [30]. Shoot-P, root-P, and seed-P are good indicators of $\mathrm{P}$ acquisition efficiency (PAE). The variations observed for shoot-P, root- $\mathrm{P}$ and seed-P indicated the genotypes differed in their ability to acquire $\mathrm{P}$ in the soils of varying levels of P. Variations observed for root biomass indicated limited soil $\mathrm{P}$ modified root architecture [31]. When plants are exposed to P-deficiency, plants start to partition photosynthates to the roots, and a decrease in shoot:root biomass ratios occurs [32]. From our results, shoot biomass was positively correlated to root biomass, shoot-P and PUE indicating that shoot biomass is an important trait for adaptation to low $\mathrm{P}$ availability. As shoot biomass increases, the root biomass also increases promoting more roots for soil exploration and thus more P uptake [33] [34]. However, the shoot:root ratio in this study was likely confounded by limited root growth due to pot size or other mechanisms important for conferring tolerance to P-deficiency in beans besides increasing root production.

To improve internal $\mathrm{P}$ utilization efficiency, it is crucial to increase shoot biomass production per unit of $\mathrm{P}$ in shoots referred to as PUE. Higher PUE is important for bean production under low soil P. PUE was positively correlated with shoot biomass, root biomass and shoot $\mathrm{P}$, and it was negatively correlated with shoot $\mathrm{P}$ concentration suggesting that when more $\mathrm{P}$ is available in the system the less efficient the genotype will be. For the low $\mathrm{P}$ treatment, Bukoba, Montcalm and Kijivu had the highest PUE scores indicating these genotypes were efficient in utilizing $\mathrm{P}$ for shoot biomass growth, and may be useful for breeding elite bean varieties tolerant to soil $\mathrm{P}$ deficiency in the future.

\section{Conclusion}

There was variation in response to low $\mathrm{P}$ availability among genotypes. Genotypes with high PUE, shoot and root biomass under limited P supply are desired, given they translocate $\mathrm{P}$ toward seed production. Some genotypes exhibited some of these qualities but not all of them. Montcalm responded well to added $\mathrm{P}$ and was more efficient in acquisition and utilization of $\mathrm{P}$ under $\mathrm{P}$ limited conditions. Conversely, G122 was the least efficient. Bukoba had high PUE but low internal $\mathrm{P}$ concentrations in the roots and shoots. Interestingly, both landrace cultivars Kijivu and Bukoba, repeatedly exposed to low $\mathrm{P}$ conditions in Tanza- 
nia, exhibited high PUE in this study. Field studies are needed to examine PAE and confirm PUE for these genotypes, as a genotype that can more effectively acquire and utilize available $\mathrm{P}$ under limited availability of this nutrient is desired.

\section{Conflicts of Interest}

The authors declare no conflicts of interest regarding the publication of this paper.

\section{References}

[1] Greenwood, D.J., Stellacci, A.M., Meacham, M.C., Broadley, M.R. and White, P.J. (2005) Phosphorus Response Components of Different Brassica oleracea Genotypes Are Reproducible in Different Environments. Crop Science, 45, 1728-1735. https://doi.org/10.2135/cropsci2004.0484

[2] Chaudhary, M.I., Gyamfi, J.J.A., Saneoka, H., et al. (2008) The Effect of Phosphorus Deficiency on Nutrient Uptake, Nitrogen Fixation and Photosynthetic Rate in Mashbean, Mungbean and Soybean. Acta Physiologiae Plantarum, 30, 537. https://doi.org/10.1007/s11738-008-0152-8

[3] Mourice, S.K. and Tryphone, G.M. (2012) Evaluation of Common Bean (Phaseolus vulgaris L.) Genotypes for Adaptation to Low Phosphorus. International Scholarly Research Network ISRN Agronomy, 2012, Article ID: 309614.

[4] Vance, C.P. (2001) Symbiotic Nitrogen Fixation and Phosphorus Acquisition. Plant Nutrition in a World of declining Renewable Resources. Plant Physiology, 127, 390-397. https://doi.org/10.1104/pp.010331

[5] Fawole, I., Gabelman, W.H. and Gerloff, G.C. (1982a) Genetic Control of Root Development in Beans (Phaseolus vulgaris L.) Grown under Phosphorus Stress. American Society for Horticultural Science, 107, 98-100.

[6] Fawole, I., Gabelman, W.H., Gerloff, G.C. and Nordheim, E.V. (1982b) Heritability of Efficiency of Phosphorus Utilization in Beans (Phaseolus vulgaris L.) Grown Under Phosphorus Stress. American Society for Horticultural Science, 107, 94-97.

[7] Singh, S.P., Urea, C.A., Gutierrez, J.A. and Garcia, J. (1989) Selection for Yield at Two Fertility Levels in Small Seeded Common Bean. Plant Science, 69, 1011-1017.

[8] Beebe, S.E., Rojas-Pierce, M., Yan, X., Blair, M.W. and Pedraza, F. (2006) Quantitative Trait Loci for Root Architecture Traits Correlated with Phosphorus Acquisition in Common Bean. Crop Science, 46, 413-423. https://doi.org/10.2135/cropsci2005.0226

[9] Vance, C.P., Stone, C.U. and Allan, D.L. (2003) Phosphorus Acquisition and Use: Critical Adaptations by Plants for Securing a Non-Renewable Resource. New Phytologist, 157, 423-447. https://doi.org/10.1046/j.1469-8137.2003.00695.x

[10] Vandamme, E., Saito, R.T., Jeong, K.K. and Wissuwa, M. (2016) Integration of P Acquisition Efficiency, P Utilization Efficiency and low Grain P Concentrations into P-Efficient Rice Genotypes for Specific Target Environments. Nutrient Cycling in Agroecosystems, 104, 413. https://doi.org/10.1007/s10705-015-9716-3

[11] Rao, I.M., Friesen, D.K. and Osaki, M. (1999) Plant Adaptation to Phosphorus-Limited Tropical Soils: In: Pessarakli, M., Ed., Handbook of Plant and Crop Stress, 2nd Edition, Marcel Dekker, New York, 61-95. 
[12] Lynch, J.P. and Beebe, S.E. (1995) Adaptation of Beans (Phaseolus vulgaris L.) to Low Phosphorus Availability. Horticultural Science, 30, 1165-1171.

[13] Bonser, A.M., Lynch, J. and Snapp, S. (1996) Effect of Phosphorus Deficiency on Growth Angle of Basal Roots in Phaseolus vulgaris. New Phytologist, 132, 281-288. https://doi.org/10.1111/j.1469-8137.1996.tb01847.x

[14] Cichy, K.A., Porch, T.G., Beaver, J.S., Cregan, P., Fourie, D., Glahn, R.P., Grusak, M.A., Kamfwa, K., Katuuramu, D.N., McClean, P., Mndolwa, E., Msolla, S.N., Corrales, M.A.P. and Miklas, P.N. (2015) A Phaseolus vulgaris Diversity Panel for Andean Bean Improvement. Crop Science, 55, 2149-2160. https://doi.org/10.2135/cropsci2014.09.0653

[15] Larsen, R.C. and Miklas, P.N. (2004) Generation and Molecular Mapping of a SCAR Marker Linked with the Bct Gene for Resistance to Beet Curly Top Virus in Common Bean. Phytopathology, 94, 320-325. https://doi.org/10.1094/PHYTO.2004.94.4.320

[16] Larsen, R.C., Druffel, K.L. and Wyatt, S.D. (2011) The Complete Nucleotide Sequences of Bean Common Mosaic Necrosis Virus Strains NL-5, NL-8 and TN-1. Archives of Virology, 156, 729-732. https://doi.org/10.1007/s00705-011-0945-8

[17] Baiges, S., Beaver, J.S., Miklas, P.N. and Rosas, J.C. (1996) Evaluation and Selection of Dry Beans for Heat Tolerance. Annual Report of the Bean Improvement Cooperative, 39, 88-89.

[18] Tock, A.J., Fourie, D., Walley, P.G., Holub, E.B., Soler, A., Cichy, K.A., Pastor-Corrales, M.A., Song, Q., Porch, T.G., Hart, J.P., Vasconcellos, R.C.C., Vicente, J.G., Barker, G.C. and Miklas, P.N. (2017) Genome-Wide Linkage and Association Mapping of Halo Blight Resistance in Common Bean to Race 6 of the Globally Important Bacterial Pathogens. Frontiers in Plant Science, 8, 1170. https://doi.org/10.3389/fpls.2017.01170

[19] Bray, R.H. and Kurtz, L.T. (1945) Determination of Organic and Available Forms of Phosphorus in Soils. Soil Science, 59, 39-45. https://doi.org/10.1097/00010694-194501000-00006

[20] Isaac, R.A. and Johnson, W.C. (1975) Collaborative Study of Wet and Dry Ashing Techniques for the Elemental Analysis of Plant Tissue by Atomic Absorption Spectrophotometry. Journal-Association of Official Analytical Chemists, 58, 436-440.

[21] White, P.J., Broadley, M.R., Greenwood, D.J. and Hammond, J.P. (2005) Genetic Modifications to Improve Phosphorus Acquisition by Roots. International Fertilizer Society, York.

[22] White, P.J. and Hammond, J.P. (2008) Phosphorus Nutrition of Terrestrial Plants. In: White, P.J. and Hammond, J.P., Eds., The Ecophysiology of Plant-Phosphorus Interactions, Springer, Dordrecht, 51-81.

[23] Schachtman, D.P., Reid, J.R. and Ayling, S.M. (1998) Phosphorus Uptake by Plants: From Soil to Cell. Plant Physiology, 116, 447-453. https://doi.org/10.1104/pp.116.2.447

[24] Minnesota North Dakota (2014) Handbook for Better Edible Bean Production. ADM Edible Bean Specialties, Inc.

[25] Fageria, N.K., Baligar, V.C. and Jones, C.A. (1997) Growth and Mineral Nutrition of Field Crops. 2nd Edition, Marcel Dekker, New York.

[26] Molaaldoila, Y.A.A. and Hakimi, K.A.A. (2016) Agronomical and Physiological Response of Common Bean (Phaseolus vulgaris L.) Genotypes to Low Soil Fertility at the Southern Highland Region of Yemen. Journal of Agricultural Science, 8. 
[27] Jones, C.A. (1983) A Survey of the Variability in Tissue Nitrogen and Phosphorus Concentrations in Maize and Grain Sorghum. Field Crops Research, 6, 133-147. https://doi.org/10.1016/0378-4290(83)90053-9

[28] Marschner, P. (2012) Mineral Nutrition of Higher Plants. Academic Press, San Diego.

[29] Yan, X.L., Beebe, S.E. and Lynch, J.P. (1995) Genetic Variation for Phosphorus Efficiency of Common Bean in Contrasting Soil Types: II. Yield Response. Crop Science, 35, 1094-1099. https://doi.org/10.2135/cropsci1995.0011183X003500040029x

[30] Wortman, C.S., Lunze, L., Ochwoh, V.A. and Lynch, J.P. (1995) Bean Improvement for Low Soil Fertility Soils in Africa. African Crop Science Journal, 3, 469-477.

[31] Miller, C.R., Ochoa, I., Nielsen, K.L., Beck, D. and Lynch, J.P. (2003) Genetic Variation for Adventitious Rooting in Response to Low Phosphorus Availability: Potential Utility for Phosphorus Acquisition from Stratified Soils. Functional Plant Biology, 30, 973-985. https://doi.org/10.1071/FP03078

[32] Marschner, H. (1995) Mineral Nutrition of Higher Plants. 2nd Edition, Academic Press, London, 889.

[33] Zhu, J. and Lynch, J.L. (2004) The Contribution of Lateral Rooting to Phosphorus Acquisition Efficiency in Maize (Zea mays L.) Seedlings. Functional Plant Biology, 31, 949-958. https://doi.org/10.1071/FP04046

[34] Liao, H., Rubio, G., Yan, X., Cao, A., Brown, K.M. and Lynch, J.P. (2001) Effect of Phosphorus Availability on Basal Root Shallowness in the Common Bean. Plant Soil, 232, 69-79. https://doi.org/10.1023/A:1010381919003 